\title{
Changes in the size of cell nuclei in response to SMC proteins degradation in mammalian cells
}

\author{
Myakinkov I. ${ }^{1,2 *}$, Khabarova A. ${ }^{2}$, Yunusova A. ${ }^{2}$, Battulin N. ${ }^{2}$ \\ ${ }^{1}$ Novosibirsk State University, Novosibirsk, Russia \\ ${ }^{2}$ Institute of Cytology and Genetics, SB RAS, Novosibirsk, Russia \\ *i.myakinkov@g.nsu.ru
}

Key words: cell nucleus size, SMC, chromatin, condensin, cohesin

Motivation and Aim: Structural maintenance of chromosomes (SMC) proteins which form condensin and cohesin complexes are crucial for the three-dimensional organization of the animal genome and cell division $[1,2]$. Besides, disruption of their work can affect the shape and size of an interphase cell nucleus while the ploidy remains unaltered [3]. We have depleted SMC complexes in human and mouse cells to determine how this affects the size of the cell nucleus and how significant the change is.

Methods and Algorithms: Three experiments were performed on cell cultures of mouse embryonic stem cells (mESC) and human HAP1 cells with modified proteins SMC2 and Rad21, parts of condensin and cohesin complexes respectively. In these cells, the proteins named above are fused with an auxin-inducible degron system. Cell nuclei with auxin added were observed after different periods, stained with DAPI, and captured on a fluorescence imaging system. Cells without auxin added took the role of the negative control. The area of the projection of the nucleus was calculated using the ImageJ program with $40-100$ nuclei taken in the sample. The data was visualized using a violin plot on the Plotly online graph maker.

Results: According to the data, an increase in the area of nuclei is observed: the mean value increases with time, reaching a maximum value 24 hours after the addition of auxin, ranging from 132 to $247 \%$ of the original projection area. Rates of increase in size during the degradation of the SMC2 protein and decay of condensins are similar in both mouse embryonic stem cells and human HAP1 cells.

Conclusion: Obtained results confirm the fact of a significant increase in the size of cell nucleus in response to the decomposition of condensin and cohesin complexes. Since reasons for this phenomenon remain enigmatic, its further study is of great interest and may help to discover new mechanisms of the cell nucleus structural regulation.

\section{References}

1. Gibcus J.H., Samejima K., Goloborodko A. et al. A pathway for mitotic chromosome formation. Science. 2018;359:6376.

2. Hoencamp C., Dudchenko O., Elbatsh A.M.O. et al. 3D genomics across the tree of life reveals condensing II as a determinant of architecture type. Science. 2021;372(6545):984-989.

3. Fazzio T.G., Panning B. Condensin complexes regulate mitotic progression and interphase chromatin structure in embryonic stem cells. J. Cell Biol. 2010;188(4):491-503. 